\title{
Renormalization of Nonequilibrium Systems with Critical Stationary States
}

\author{
Alessandro Vespignani, ${ }^{1}$ Stefano Zapperi, ${ }^{2}$ and Vittorio Loreto ${ }^{3}$ \\ ${ }^{1}$ Instituut-Lorentz, University of Leiden, P.O. Box 9506, 2300 RA, Leiden, The Netherlands \\ ${ }^{2}$ Center for Polymer Studies and Department of Physics, Boston University, Boston, Massachusetts 02215 \\ ${ }^{3}$ ENEA Research Center, loc. Granatello, C.P.32, 80055 Portici, Napoli, Italy
}

(Received 15 May 1996)

\begin{abstract}
We introduce the general formulation of a renormalization method suitable to study the critical properties of nonequilibrium systems with steady states: the dynamically driven renormalization group. We renormalize the time evolution operator by computing the rescaled time transition rate between coarse grained states. The obtained renormalization equations are coupled to a stationarity condition which provides the approximate nonequilibrium statistical weights of steady-state configurations to be used in the calculations. In this way we are able to write recursion relations for the parameter evolution under scale change, from which we can extract numerical values for the critical exponents. This general framework allows the systematic analysis of several models showing self-organized criticality in terms of usual concepts of phase transitions and critical phenomena. [S0031-9007(96)01709-7]

PACS numbers: 64.60.Ak, 05.40.+j, 64.60.Lx
\end{abstract}

In the last decade nonequilibrium critical phenomena have attracted a wide interest in statistical physics. Critical systems are characterized by the absence of a characteristic lengthscale, strong fluctuations, and nonanalyticity of the correlation functions. Examples of this behavior can be found in phase transitions [1-3], self-organized critical (SOC) systems [4], fractal growth [5], and a vast class of complex systems [6]. The major source of difficulties in the study of nonequilibrium critical phenomena $[3,7]$ lies in the absence of a general criterion, like the use of the Gibbs distribution in equilibrium systems, to assign an ensemble statistical measure to a particular configuration of the system. The probability distribution is instead a time dependent solution of a master equation, which only in some particular cases becomes stationary in the long time limit.

In this Letter we present the general formalism of a real space dynamical renormalization group (RG) scheme for systems with a nonequilibrium critical steady state: the dynamically driven renormalization group (DDRG). The method combines the renormalization of the time evolution operator with a stationarity condition which allows the calculation of the approximate steady-state configurations probability distribution. This coupling acts at each coarse graining step and therefore represents a driving for the renormalization group equations. For SOC systems [8-10], the DDRG allows us to derive in a broader framework previous RG schemes [11-13] and to formulate a more systematic approach. Here we show the explicit application of the DDRG to the forest-fire model (FFM) $[9,10]$, which we can now study in the whole parameters space. Possible applications of the DDRG are not restricted to SOC models: The method can be used to study other equilibrium or nonequilibrium critical phenomena such as driven diffusive systems [2,3], which to our knowledge have never been approached by real space RG methods.
We consider discrete lattice models on a $d$-dimensional lattice. To each site $i$ is associated a variable $\sigma_{i}$, which can assume $q$ different values $\left(\sigma_{i}=0,1, \ldots, q\right)$. A complete set $\sigma \equiv\left\{\sigma_{i}\right\}$ of lattice variables specifies a configuration of the system. We define $\left\langle\sigma|T(\mu)| \sigma^{0}\right\rangle$ as the transition rate from a configuration $\sigma_{0}$ to a configuration $\sigma$ in a time step $t$ as a function of a set of parameters $\mu=\left\{\mu_{i}\right\}$. The time dependent probability distribution $P(\sigma, t)$ for the configurations of the system obeys the following master equation (ME):

$$
P\left(\sigma, t_{0}+t\right)=\sum_{\left\{\sigma^{0}\right\}}\left\langle\sigma|T(\mu)| \sigma^{0}\right\rangle P\left(\sigma^{0}, t_{0}\right) .
$$

The explicit solution of the master equation is in general not available, but we can extract the critical properties of the model by a renormalization group analysis. We coarse grain the system by rescaling lengths and time according to the transformation $x \longrightarrow b x$ and $t \longrightarrow b^{z} t$. The renormalization transformation is constructed through an operator $\mathcal{R}(S, \sigma)$ that introduces a set of coarse grained variables $S \equiv\left\{S_{i}\right\}$ and rescales the lengths of the system [14]. In general, $\mathcal{R}$ is a projection operator with the properties $\mathcal{R}(S, \sigma) \geq 0$ for any $\left\{S_{i}\right\},\left\{\sigma_{i}\right\}$, and $\sum_{\{S\}} \mathcal{R}(S, \sigma)=1$. These properties preserve the normalization condition of the renormalized distribution. The explicit form of the operator $\mathcal{R}$ is defined case by case in the various applications of the method. Usually it corresponds to a block transformation in which lattice sites are grouped together in a super-site that defines the renormalized variables $S_{i}$ by means of a majority or spanning rule.

We subdivide the time step in intervals of the unitary time scale $\left(t_{0}=0\right)$ obtaining the coarse graining of the system as follows:

$$
P^{\prime}\left(S, t^{\prime}\right)=\sum_{\{\sigma\}} \mathcal{R}(S, \sigma) \sum_{\left\{\sigma^{0}\right\}}\left\langle\sigma\left|T^{b^{z}}(\mu)\right| \sigma^{0}\right\rangle P\left(\sigma^{0}, 0\right),
$$


where we have included the application of the operator $\mathcal{R}$ and $t^{\prime}=b^{z} t$. The meaning of $\left\langle\sigma\left|T^{b^{z}}(\mu)\right| \sigma^{0}\right\rangle$ has to be defined explicitly: The simplest possibility is $b^{z}=$ $N$ where $N$ is an integer number, and $T^{N}$ denotes the application of the dynamical operator $N$ times. In general, since we are dealing with a discrete time evolution we have to consider $T^{b^{2}}$ as a convolution over different paths, chosen by an appropriate condition. The detailed definition of the effective operator $T^{b^{2}}$ is reported in Ref. [15]. By multiplying and dividing each term of Eq. (2) by $P^{\prime}\left(S^{0}, 0\right)=\sum_{\left\{\sigma^{0}\right\}} \mathcal{R}\left(S^{0}, \sigma^{0}\right) P\left(\sigma^{0}, 0\right)$ and using the properties of the operator $\mathcal{R}$, we get, after some algebra,

$$
P^{\prime}\left(S, t^{\prime}\right)=\sum_{\left\{S^{0}\right\}}\left[\frac{\sum_{\left\{\sigma^{0}\right\}} \sum_{\{\sigma\}} \mathcal{R}\left(S^{0}, \sigma^{0}\right) \mathcal{R}(S, \sigma)\left\langle\sigma\left|T^{b^{2}}(\mu)\right| \sigma^{0}\right\rangle P\left(\sigma^{0}, 0\right)}{\sum_{\left\{\sigma^{0}\right\}} \mathcal{R}\left(S^{0}, \sigma^{0}\right) P\left(\sigma^{0}, 0\right)}\right] P^{\prime}\left(S^{0}, 0\right),
$$

which finally identifies the renormalized dynamical operator $\left\langle S\left|T^{\prime}\right| S^{0}\right\rangle$. In other words the new dynamical operator $T^{\prime}$ is the sum over all the dynamical paths of $b^{z}$ steps that from a starting configuration $\left\{\sigma_{i}^{0}\right\}$ lead to a configuration $\left\{\sigma_{i}\right\}$, which renormalize, respectively, in $\left\{S_{i}^{0}\right\}$ and $\left\{S_{i}\right\}$. The sum is weighted by the normalized statistical distribution of each configuration.

We apply this scheme to systems with a steady state described by a stationary distribution $P(\sigma, t \longrightarrow \infty)=$ $W(\sigma)$. For equilibrium systems the stationary distribution has the Gibbs form $W(\sigma) \sim \exp [-\beta H(\sigma)]$, where $H(\sigma)$ is the Hamiltonian. There is not such a general criterion for nonequilibrium dynamical systems, therefore we have developed an approximate method to evaluate the stationary distribution to be used in the calculation of the renormalized master equation. The simplest approximation considers only the incoherent part of the stationary distribution which does not include correlations and can therefore be factorized. For systems characterized by $q$-state variables it has the form

$$
W^{(i)}(\sigma)=\prod_{i}\left\langle\rho_{\sigma_{i}}\right\rangle,
$$

where $\left\langle\rho_{\kappa}\right\rangle$ is the average density of sites in the $\kappa$ state. In this way, we have approximated the probability of each configuration $\left\{\sigma_{i}\right\}$ as the product measure of the mean field probability to have a state $\sigma_{i}$ in each corresponding site. The values of the densities $\left\{\left\langle\rho_{\kappa}\right\rangle\right\}$ as a function of the parameters $\mu$ are obtained by solving appropriate meanfield equations in the long time limit. These equations have the form of a stationarity condition

$$
\frac{\partial}{\partial t}\left\{\left\langle\rho_{\kappa}\right\rangle\right\}=S_{\mu}\left(\left\{\left\langle\rho_{\kappa}\right\rangle\right\}\right)=0,
$$

where the operator $S_{\mu}$ describes the evolution of the system as a function of the dynamical parameters defined above. Time independent solutions of Eq. (5) will be referred to as "steady states," although we should keep in mind that those are only the average states of the ensemble [16]. In ordinary statistical systems, Eq. (5) represents the thermodynamic equilibrium condition. For driven dynamical systems, it describes the driving of the system to the nonequilibrium steady state, by means of a balance condition.

By inserting this approximate distribution in Eq. (3), we obtain the renormalized dynamical operator

$$
\left\langle S\left|T^{\prime}(\mu)\right| S^{0}\right\rangle=\frac{\sum_{\left\{\sigma^{0}\right\}} \sum_{\{\sigma\}} \mathcal{R}\left(S^{0}, \sigma^{0}\right) \mathcal{R}(S, \sigma)\left\langle\sigma\left|T^{b^{2}}(\mu)\right| \sigma^{0}\right\rangle \prod_{i}\left\langle\rho_{\sigma_{i}^{0}}\right\rangle}{\sum_{\left\{\sigma^{0}\right\}} \mathcal{R}\left(S^{0}, \sigma^{0}\right) \prod_{i}\left\langle\rho_{\sigma_{i}^{0}}\right\rangle},
$$

where the densities are calculated at each coarse graining step from the stationary condition [Eq. (5)] with the corresponding renormalized dynamical parameters $\{\mu\}$. Since in this framework Eq. (5) drives the RG equations acting as a feedback on the scale transformation, we call it the driving condition.

Equations (5) and (6) are the basic renormalization equations from which the desired recursion relations are obtained. Imposing that the renormalized operator $T^{\prime}$ has the same functional form of the operator $T$, i.e., $T^{\prime}(\mu)=T\left(\mu^{\prime}\right)$, we obtain the rescaled parameter set $\mu^{\prime}=$ $f(\mu)$. This implies that the renormalized single time distribution $P^{\prime}\left(S, t^{\prime}\right)$ has the same functional form of the original distribution $P(\sigma, t)$. The critical behavior of the model is obtained by studying the fixed points $\mu^{*}=f\left(\mu^{*}\right)$. Since we are dealing with discrete evolution operators $T$, we define the time scaling factor $b^{z}$ as the average number of steps we apply the operator $T$ in order to obtain that $T^{\prime}(\mu)=T\left(\mu^{\prime}\right)$ for the coarse grained system. In this way we obtain a time recursion relation $t^{\prime}=g(\mu) t$, or equivalently $b^{z}=g(\mu)$, from which it is possible to calculate the dynamical critical exponent $z=\ln g\left(\mu^{*}\right) / \ln b$. In this form of the DDRG, we take into account only the uncorrelated part of the steadystate probability distribution. The results obtained are not trivial because correlations in the systems are considered in the dynamical renormalization of the operator $T$, that given a starting configuration traces all the possible paths leading to the renormalized final configuration. Moreover, geometrical correlations are treated by the operator $\mathcal{R}$ that maps the system by means of spanning conditions or majority rules. The renormalized uncorrelated part of the stationary distribution is evaluated from the stationary condition with renormalized parameters, thus providing an 
effective treatment of correlations. One can then improve the results by including higher order contributions to the unknown stationary distribution $W(\sigma)$ using cluster variation methods [17]. Naturally the above scheme can also be applied to equilibrium critical phenomena, where the driving condition is represented by the equilibrium mean field equations [15].

The DDRG is a useful tool to study the critical properties of SOC systems. In fact, these systems evolve spontaneously in a scale invariant stationary state. The forest-fire model is a simple automaton which has been introduced by Bak et al. [9] as an example of SOC, and has been then modified by Drossel and Schwabl [10]. The model is defined on a lattice in which each site can be empty $\left(\sigma_{i}=0\right)$, occupied by a green tree $\left(\sigma_{i}=1\right)$ or by a burning tree $\left(\sigma_{i}=2\right)$. At each time step the lattice is updated as follows: (i) A burning tree becomes an empty site; (ii) a green tree becomes a burning tree if at least one of its neighbors is burning; (iii) a tree can grow in an empty site with probability $p$; (iv) a tree without burning nearest neighbors becomes a burning tree with probability $f$. The model was first studied in the case $f=0$ for the limit of very slow tree growth $(p \longrightarrow 0)$. In this limit the critical behavior is trivial: The model shows spiral-shaped fire fronts separated by a diverging length $\xi \sim p^{-\nu_{p}}$, where $\nu_{p} \simeq 1$ [18]. In the case $f>0$, the system is supposed to exhibit SOC under the hypothesis of a double separation of time scales: Trees grow fast compared with the occurrence of lightnings and forest clusters burn down much faster than trees grow. This request is expressed by the double limit $\theta \equiv f / p \rightarrow 0$ and $p \rightarrow 0$. The critical state is characterized by a power law distribution $P(s)=s^{-\tau}$ of the forest clusters of $s$ sites (avalanches in the SOC terminology) and the average cluster radius (the correlation length) scales as $R \sim \theta^{-\nu_{R}}$.

With the DDRG framework we are able to generalize a previous RG scheme [12] in order to include the proper treatment of the time scaling and to study the limit $f=0$ (deterministic FFM). The dynamical rules of the FFM are local and the set of dynamical parameters, defined by $\mu=\{f, p\}$, is obtained explicitly in terms of the dynamical operators acting on a single site, i.e., $\langle 1|T| 0\rangle=p$ and $\langle 2|T| 1\rangle=f$. The relevant dynamical scales is defined by the burning process which occurs with probability one. We define a cell-to-site transformation with scale factor $b=2$ or larger. The rules defining the cell renormalization operator $\mathcal{R}$ are standard geometrical spanning conditions [19], and their explicit form can be found in Ref. [15]. The above scheme defines a finite lattice truncation on four (two) sites cells in $d=2$ $(d=1)$, and denoting by an index $\alpha$ each cell configuration, we have that $\sum_{\left\{\sigma_{i}\right\}} \longrightarrow \sum_{\alpha}$. The renormalization equations that define the renormalized parameters can be conveniently written as

$$
\left\langle S_{i}\left|T^{\prime}\right| S_{i}^{0}\right\rangle=\frac{\sum_{\alpha} \sum_{\alpha^{\prime}}\left\langle\alpha^{\prime}\left|T^{b^{z}}\right| \alpha\right\rangle W_{\alpha}}{\sum_{\alpha} W_{\alpha}},
$$

where $|\alpha\rangle$ and $\left|\alpha^{\prime}\right\rangle$ are the cell states which renormalize, respectively, in $\left|S_{i}^{0}\right\rangle$ and $\left|S_{i}\right\rangle$. We keep the subscript $i$ since the states refer now to a single coarse grained site and not to a configuration of the system. With $W_{\alpha}$ we denote the stationary statistical weight of each $\alpha$ configuration. This distribution is approximate following the DDRG scheme in the lowest order [Eq. (4)], in which the average steady-state densities $\left\langle\rho_{\kappa}\right\rangle$ are obtained as a function of $\mu=\{f, p\}$ from the stationary solution of dynamical mean field equations [20].

We focus our analysis in the critical region denoted by the condition $f \ll p \ll 1$, namely where the system shows critical behavior. The time scaling factor is obtained by imposing that the renormalized burning process occurs with probability one $\left(\left\langle 0\left|T^{\prime}\right| 2\right\rangle=1\right)$. In $d=1$ this condition is fulfilled up to second order in $f$ and $p$ and gives $z=1$, recovering the exact result of Ref. [21]. This result is due to the fact that in $d=1$ there is only a possible way to span the cell, and consequently no proliferations are generated. In $d=2$ one has to consider the average over different paths, and new dynamical interactions are generated at each RG step. This is a signature that we need an approximation which truncates the parameter space after each iteration so that it remains closed. This is done by considering just the leading order in $f$ and $p$ in the renormalization equations, and ignoring any proliferations generated at each group iteration. With this scheme we obtain $z=1$, which is not an exact result also if in good agreement with numerical simulations $(z=1.04$ [22]). It is worth remarking that the DDRG allows one to overcome the approximations present in the approach of Ref. [12], where the time scaling was not properly considered because of the assumption of an infinite time scale separation. In addition the general scheme shown so far provides the inroad towards a systematic improvement of the results by introducing higher order correlations in the stationary distribution as discussed in Ref. [23].

Once the time scale factor is set we can write recursion relations for $p$ and $f$, or equivalently $\theta^{\prime}=x(\theta, p)$ and $p^{\prime}=y(\theta, p)$, evaluating the probabilities that a coarse grained cell grows or is struck by a lightning in $b^{z}$ steps. The driving condition and recursion relations derivation is long and tedious and the explicit equations are reported elsewhere [15]. The flow diagram is stable with respect to different coarse graining rules, and for $d=1$ and $d=2$ we find a repulsive fixed point in $\theta_{c}=0$ and $p_{c}=0$. The fixed point densities are obtained from the driving condition and depend on the dimensionality. In order to discuss the critical behavior we have to linearize the recursion relations in the proximity of this fixed point and to find the relevant eigenvalues of the diagonal transformation:

$$
\lambda_{1}=\left.\frac{\partial \theta^{\prime}}{\partial \theta}\right|_{\theta_{c}, p_{c}}, \quad \lambda_{2}=\left.\frac{\partial p^{\prime}}{\partial p}\right|_{\theta_{c}, p_{c}} .
$$


In $d=2$ the largest eigenvalue is given by $\lambda_{1}$, which determines the leading scaling exponent $\nu_{R}=\ln b / \ln \lambda_{1}=$ 0.7 (for $b=2$ ) obtained in Ref. [12]. The result is in good agreement with numerical simulation $\left(\nu_{R}=0.6\right)$ [22]. In the limit $f=0$ the critical behavior is governed by the second eigenvalue $\lambda_{2}$. This eigenvalue and its relative exponent describes the behavior of the correlation length in the deterministic FFM. As opposed to $\lambda_{1}$, the value of $\lambda_{2}$ depends on the absolute value of the time scaling factor [24], and therefore could not be obtained without the DDRG formalism. The numerical value we obtain in $d=1,2$ is $\nu_{p}=\ln 2 / \ln \lambda_{2}=1.0$, which is in excellent agreement with the simulation results $\nu_{p} \simeq 1$ [18].

Our characterization of the flow diagram clarifies the critical nature of the model. The FFM is critical only for $\theta_{c}=0, p_{c}=0$. This implies that $\theta, p$ are the control parameters of the model, and the critical state is reached only by a fine tuning of these parameters. Similar results are obtained by applying the DDRG to the sandpile model [15]. These results allow us to clarify the meaning of SOC with respect to nonequilibrium critical phenomena. In SOC literature it is often reported that the origin of scale invariance in nature lies in the absence of tuning parameter, like the critical temperature in Ising models. In the renormalization group language this would imply that no relevant parameters should be present. The situation is, however, more subtle. It has been recognized that a common characteristic of SOC systems is the presence of two time scales $\tau_{a}$, the typical relaxation (activity) time, and $\tau_{d}$ the external driving time scale (often an external noise). In order to observe criticality the ratio $\mathcal{T}=$ $\tau_{a} / \tau_{d}$ must be vanishingly small $(\mathcal{T} \longrightarrow 0)$ [24,25]. With our approach we can recast the above concept in more formal terms. Our RG analysis shows the time scales ratio $\mathcal{T}$ is indeed the control parameter of SOC models. This parameter is the ratio between $f, p$, and the burning time scale in the forest-fire model or the sand addition and the avalanche dissipation in sandpiles, but is always related to the ratio between different time scales. From a theoretical point of view the critical nature of SOC systems is not different from that of nonequilibrium phase transitions. The peculiarity of these systems is that close to the critical point the system is quite stable to changes of the dynamical time scales. In fact, the reduced control parameter, which is defined as $\epsilon=\left(\mathcal{T}-\mathcal{T}_{c}\right) / \mathcal{T}_{c}$, in SOC systems is $\mathcal{T}$ itself, being $\mathcal{T}_{c}=0$. This implies that if $\epsilon \simeq 0$, even relevant changes of the control parameter $\left(\mathcal{T} \longrightarrow n \mathcal{T}\right.$ and $\left.n<\epsilon^{-1}\right)$ do not drive the system far from the critical region. Apparently the system would not be affected by changes of $\mathcal{T}$, and in this sense SOC systems are not very sensitive to fine tuning of the control parameter. The meaning of SOC is then related to the widespread existence of phenomena ruled by very different time scales and not to the absence of relevant control parameters as often reported in the literature.
A. V. is indebted with J.M. J. van Leeuwen for very interesting discussions. The Center for Polymer Studies is supported by the NSF.

[1] Phase Transition and Critical Phenomena, edited by C. Domb and M.S. Green (Academic Press, London, 1972-1976), Vols. 1-6; Phase Transition and Critical Phenomena, edited by C. Domb and J.L. Lebowitz (Academic Press, London, 1983-1995), Vols. 7-17.

[2] S. Katz, J. L. Lebowitz, and H. Spohn, Phys. Rev. B 28, 1655 (1983); J. Stat. Phys. 34, 497 (1984).

[3] B. Schmittmann and R. K. Zia, in Ref. [1], Vol. 17.

[4] For a review, see, e.g., P. Bak and M. Creutz, in Fractals and Disordered Systems, edited by A. Bunde and S. Havlin (Springer-Verlag, Heidelberg, 1993), Vol. II.

[5] T. Vicsek, Fractal Growth Phenomena (World Scientific, Singapore, 1992).

[6] B. B. Mandelbrot, The Fractal Geometry of Nature (Freeman and Company, New York, 1983).

[7] A. Erzan, L. Pietronero, and A. Vespignani, Rev. Mod. Phys. 67, 545 (1995).

[8] P. Bak, C. Tang, and K. Wiesenfeld, Phys. Rev. Lett. 59, 381 (1987); Phys. Rev. A 38, 364 (1988).

[9] P. Bak, K. Chen, and C. Tang, Phys. Lett. A 147, 297 (1990).

[10] B. Drossel and F. Schwabl, Phys. Rev. Lett. 69, 1629 (1992).

[11] L. Pietronero, A. Vespignani, and S. Zapperi, Phys. Rev. Lett. 72, 1690 (1994); A. Vespignani, S. Zapperi, and L. Pietronero, Phys. Rev. E 51, 1711 (1995).

[12] V. Loreto, L. Pietronero, A. Vespignani, and S. Zapperi, Phys. Rev. Lett. 75, 465 (1995).

[13] E. V. Ivashkevich, Phys. Rev. Lett. 76, 3368 (1996).

[14] T. Niemeijer and J. M. J. van Leeuwen, in Ref. [1], Vol. 6.

[15] A. Vespignani, S. Zapperi, and V. Loreto (to be published).

[16] J. Keizer, Statistical Thermodynamics of Nonequilibrium Processes (Springer Verlag, New York, 1987).

[17] R. Dickman, Phys. Rev. A 38, 2588 (1988).

[18] P. Grassberger and H. Kantz, J. Stat. Phys. 63, 685 (1991); W. K. Mossner, B. Drossel, and F. Schwabl, Physica (Amsterdam) 190A, 205 (1992).

[19] R. J. Creswick, H. A. Farach, and C. P. Poole, Jr., Introduction to Renormalization Group Methods in Physics (Wiley, New York, 1992).

[20] K. Christensen, H. Flyvbjerg, and Z. Olami, Phys. Rev. Lett. 71, 2737 (1993).

[21] B. Drossel, S. Clar, and F. Schwabl, Phys. Rev. Lett. 71, 3739 (1993).

[22] S. Clar, B. Drossel, and F. Schwabl, Phys. Rev. E 50, 1009 (1994).

[23] H. Patzlaff and S. Trimper, Phys. Lett. A 189, 187 (1994).

[24] B. Drossel (private communication).

[25] G. Grinstein, in Scale Invariance, Interfaces and NonEquilibrium Dynamics, edited by A. McKane et al., NATO Advanced Study Institutes, Ser. B, Vol. 344 (Plenum, New York, 1995). 